\title{
Nanoscale Torsional Resonator for Polarization and Spectroscopy of Nuclear Spins
}

\author{
Mark C. Butler, ${ }^{*}$ Valerie A. Norton, and Daniel P. Weitekamp \\ A. A. Noyes Laboratory of Chemical Physics, California Institute of Technology, \\ MC 127-72, Pasadena, California 91125, USA \\ (Received 26 March 2010; published 19 October 2010)
}

\begin{abstract}
We propose a torsional resonator that couples to the transverse spin dipole of an attached sample. The absence of relative motion eliminates a source of friction that would otherwise hinder nanoscale implementation. Enhanced spontaneous emission induced by the resonator relaxes the longitudinal spin dipole at a rate of $\sim 1 \mathrm{~s}^{-1}$ in the low-temperature limit. With signal averaging, single-proton magnetic resonance spectroscopy appears feasible at $\sim 10 \mathrm{mK}$ and a high magnetic field, while single-shot sensitivity is practical for samples with at least tens of protons in a volume of $\sim 5 \mathrm{~nm}^{3}$.
\end{abstract}

DOI: 10.1103/PhysRevLett.105.177601

Although nuclear magnetic resonance (NMR) spectroscopy is widely used as a structural probe of solids, it is generally limited by sensitivity to samples of at least the micron scale, containing at least nanomoles of the molecules of interest. Improving the detector sensitivity and increasing the fractional spin polarization $P$ are complementary approaches to improving spectrometer sensitivity. Major improvements in detector sensitivity are required in order to reduce the requisite number of nuclear spins $N$ to 1 . Because of the significance of spin noise in weakly polarized nanoscale samples, strong polarization is also desirable in order to achieve single-shot signal-to-noise ratios that are substantially greater than unity for samples with $N \gg 1$. Thus, the ideal spectrometer would have a sensitive detector and be capable of rapidly polarizing the spins to $P \sim 1$, thereby enabling NMR as a tool for studying the electronic and nuclear structure of systems such as individual biomolecules, quantum dots, defects, and atomic sites at interfaces.

The use of a magnetic mechanical oscillator as a sensitive detector of longitudinal spin magnetization has been demonstrated for imaging in a field gradient with magnetic resonance force microscopy (MRFM) [1,2], for NMR spectroscopy in a nominally homogeneous magnetic field [3,4], and for multidimensional experiments that encode spectral and spatial information during distinct portions of each transient [5]. In particular, MRFM with a resolution of $<10 \mathrm{~nm}$ has been demonstrated [2]. The ultimate sensitivity requires a low temperature to minimize thermal noise of the mechanical resonator. Practical NMR spectroscopy, especially multidimensional time-domain methods, generally depends on the acquisition of many transients, with the fractional spin polarization $P$ being restored between transients as the spins relax toward the thermal equilibrium value $P_{\text {th }}$. However, at millikelvin temperatures $T$ and high Larmor frequencies $\omega_{0}=-\gamma B_{0}$, where $P_{\mathrm{th}}=\tanh \left(-\hbar \omega_{0} / 2 k T\right)$ approaches unity, the fluctuating spin-lattice interactions which restore equilibrium become "frozen out," yielding impractically long delays
PACS numbers: 82.56. $-\mathrm{b}, 33.25 .+\mathrm{k}, 37.10 .-\mathrm{x}, 76.60 .-\mathrm{k}$

between transients [6]. As we show here, cooling a nanoscale mechanical resonator to low temperature $T_{h}$, such that the number $n_{\text {th }}$ of quanta in the resonator approaches zero, has the additional benefit of enabling a general method of rapidly polarizing the spins to $P_{\mathrm{th}} \sim 1$, eliminating the need for efficient spin-lattice relaxation.

The present resonator design is a variant of the BOOMERANG (better observation of magnetization, enhanced resolution, and no gradient) scheme $[3,4]$, which allows mechanical detection of magnetic resonance without a field gradient across the sample. In nanoscale extrapolations of both MRFM [1] and BOOMERANG [4], it has been recognized that the inverse scaling of mechanical frequency with the length scale leads to the desirability of coupling the resonator to transverse, rather than longitudinal, magnetization. A key innovation of the present design (Fig. 1) is that this is achieved with no relative motion between the sample and the magnet, allowing an arbitrarily close approach of the sample and magnet. This is accomplished by inserting the sample between ferromagnetic or paramagnetic cylinders which also "sandwich" the center of a $\mathrm{Si}$ beam suspended at both ends to create a torsional

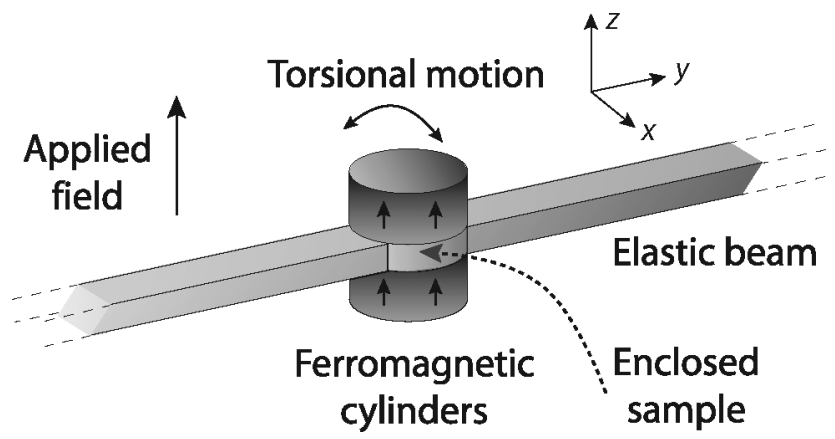

FIG. 1. Prototypical resonator design for NMR spectroscopy of nanoscale samples. The sample is "sandwiched" between magnetic cylinders and rotates with the sandwich about the torsional beam. The spin dipole couples to the oscillating transverse field generated by the cylinders. 
resonator with fundamental frequency $\omega_{h}$. The magnetic cylinders are assumed to be fully saturated along the $z$ axis by a high applied magnetic field $B_{a}$ and low lattice temperature. The sample rotates with the sandwich as the beam performs torsional oscillations, and the absence of relative motion between the sample and other moving parts eliminates an important source of surface friction that would otherwise damp the resonator and provide a fundamental limit to miniaturization. (The supplementary information [7] includes additional details concerning the resonator design and the spin-resonator coupling.)

For small mechanical motions, the field $\mathbf{B}(\theta)$ at the spins can be approximated to first order in the resonator's coordinate $\theta$, defined as the angular displacement of the sandwich as it rotates about the $y$ axis oriented along the beam. This approximation gives $B_{x}(\theta) \approx\left(d B_{x} / d \theta\right) \theta$, with $B_{y}(\theta) \approx 0$ by symmetry. The time-average field $B_{0}$ defining the quantization axis of the spins is the sum of the applied field $B_{a}$ and the field $B_{h}$ due to the sandwich magnets, and is independent of $\theta$ to first order. For a system of noninteracting spins $1 / 2$ which experience the same field, the interaction term in the lab-frame Hamiltonian governing the spin-resonator system can be expressed in units of frequency as

$$
H_{\mathrm{int}}=\left(-\gamma \frac{d B_{x}}{d \theta}\right) I_{x} \theta,
$$

where $\gamma$ is the gyromagnetic ratio and, the spin component $I_{x}$ is summed over the $N$ spin $1 / 2$ nuclei. Switching to the interaction frame and making the rotating-wave approximation at the resonance condition $\omega_{h}=-\omega_{0}$ yields

$$
\begin{gathered}
H_{1}=g\left(I_{+} a^{\dagger}+I_{-} a\right), \\
g=\frac{-\gamma}{2} \sqrt{\frac{\hbar}{2 I_{h} \omega_{h}}} \frac{d B_{x}}{d \theta}=\frac{3 \gamma B_{h}}{4} \sqrt{\frac{\hbar}{2 I_{h} \omega_{h}} .}
\end{gathered}
$$

Here $a^{\dagger}$ and $a$ are, respectively, the raising operator and lowering operator for the mechanical oscillator, $I_{+}$and $I_{-}$ are, respectively, the spin raising operator and lowering operator summed over all spins, and $I_{h}$ is the moment of inertia. The Hamiltonian $H_{1}$ has the form of the JaynesCummings Hamiltonian [8] but with a coupling parameter $g$ that describes the conversion of oscillator motion into a resonant rotating field. The second equality in Eq. (2) results from assuming that the magnetization of the sandwich cylinders remains aligned along $z$ independent of $\theta$ (soft magnet). The value of $g$ would be reduced by $2 / 3$ if instead the magnetization remained aligned along the cylinder axis (hard magnet). Equation (1) also assumes $\gamma>0$; in the case where $\gamma<0$, the interaction Hamiltonian is $H_{1}=g\left(I_{+} a+I_{-} a^{\dagger}\right)$. Regardless of the sign of $\gamma$, however, the two product operators contributing to $H_{1}$ each exchange a quantum between spins and resonator, and the phenomena of interest are similar.

A principal concern here is how the spin polarization couples to a cold resonator. If the resonator is strongly damped, in the sense that energy donated to it by the spins is dissipated into the thermal bath before cycling back to the spins, then the resonator may itself be treated as a bath that damps the spins, and the spin evolution is described by a reduced master equation that does not explicitly include the resonator's degrees of freedom [9]. By using the reduced master equation, it is straightforward to show that if a single spin interacts with a strongly damped resonator at temperature $T_{h}$, then

$$
\frac{d}{d t}\left\langle I_{z}-\left\langle I_{z}\right\rangle_{\mathrm{th}}\right\rangle=-R_{h}\left\langle I_{z}-\left\langle I_{z}\right\rangle_{\mathrm{th}}\right\rangle,
$$

which gives equilibration governed by the rate constant

$$
R_{h}=2 g^{2} \tau_{h}\left(2 n_{\text {th }}+1\right),
$$

where $\left\langle I_{z}\right\rangle_{\mathrm{th}}=(N / 2) \tanh \left(-\hbar \omega_{0} / 2 k T_{h}\right)$ is the mean value of $I_{z}$ for $N$ spins in thermal equilibrium at temperature $T_{h}$. Note that the rate constant $R_{0}=2 g^{2} \tau_{h}$ obtained by setting $n_{\mathrm{th}}=0$ can be interpreted as characterizing enhanced spontaneous emission from the spins to the resonant mechanical mode, which provides a means of spin polarization effective at an arbitrarily low temperature. Thermal excitation in the resonator, characterized by nonzero $n_{\mathrm{th}}$, results in incoherent stimulated absorption and emission by the spins, which speeds equilibration but to a higher spin temperature. Note that the high-temperature limit of the present method of spin polarization is distinct from, but related to, the mechanism by which a large field gradient was used to relax spin-locked transverse magnetization toward zero in an MRFM geometry [10].

The reduced master equation for $N$ noninteracting spins gives

$$
\frac{d}{d t}\left\langle I_{z}\right\rangle=-R_{h}\left[\left\langle I_{z}\right\rangle\left(1-P_{\mathrm{th}}\right)-\left\langle I_{-} I_{+}\right\rangle P_{\mathrm{th}}\right] .
$$

Starting from an initial condition of independent Zeeman spin ordering at non-negative spin temperature, e.g., $\left\langle I_{z}\right\rangle=0$, no mean transverse magnetization develops and the polarization begins to grow as for a single spin [Eq. (3)] but then slows and reaches a steady state which in general is less than $P_{\text {th }}$. At $T_{h}=0$, where $P_{\text {th }}=1$, further polarization is arrested when $\left\langle I_{-} I_{+}\right\rangle$vanishes due to the buildup of correlations among the spins through their interaction with the harmonic oscillator mode [11]. Since the transverse magnetization $\left\langle I_{ \pm}\right\rangle=0$ during this process, $\left\langle I_{-} I_{+}\right\rangle$ (the norm squared of the complex transverse magnetization) is a variance or noise power, which is damped in the course of creating spin order.

The reason that relaxation of the polarization cannot continue to completion solely by the coupling of the spins to the single quantum mechanical mode is that the Hamiltonian $H_{1}$ commutes with $\mathbf{I}^{2}$, so spin angular momentum is conserved during the relaxation, in the absence of other interactions that break this symmetry. A system of $N$ spins $1 / 2$ contains one angular momentum manifold with $I=N / 2$, and the ground state is the minimum-energy state of this manifold. Resonator-induced spin relaxation does not transfer population between angular momentum 
manifolds of different $I$; rather, it tends to bring each manifold into thermal equilibrium with the resonator. If the initial spin distribution has population in any of the manifolds with $I<N / 2$, and if other terms in the spin Hamiltonian do not transfer population between manifolds, then the interaction will leave the population trapped away from the ground state [11]. Starting from no spin order, the fractional polarization per spin in this trapped state for $T_{h}=0$ is

$$
P_{\text {trap }}=\frac{1}{2^{N-1} N} \sum_{J}^{N / 2} \frac{N !(2 J+1)^{2}}{\left(\frac{N}{2}+J+1\right) !\left(\frac{N}{2}-J\right) !},
$$

which is $\sim \sqrt{2 / N}$ for $N \gg 1$, so even this "worst case" relaxation scenario gives a net signal $P_{\text {trap }} N / 2 \approx \sqrt{N / 2}$, which is both larger than spin noise and has a definite sign, allowing efficient signal averaging.

The resonator-induced polarization of $N$ spins in a solid will be affected by the chemical-shift differences and the couplings between spins, since these interactions couple spin states with different total angular momentum. Simulations of resonator-induced polarization in three-proton, four-proton, and five-proton systems performed with the GAMMA C++ library [12] suggest that, as the number of spins is increased beyond 3, the dipolar coupling becomes increasingly effective in coupling manifolds of different angular momentum, thereby allowing efficient polarization toward $P_{\text {th }}$ (see [7] for relaxation plots). Figure 2 shows simulations of the relaxation of 50 spins for three idealized cases, all simulated by using $R_{h}=1 \mathrm{~s}^{-1}$ and $T_{h}=0$, with equal initial population of all states. The dashed curve is the case of isochronous spins interacting only through the resonator and cooling to $P_{\text {trap }}$. The solid curve is

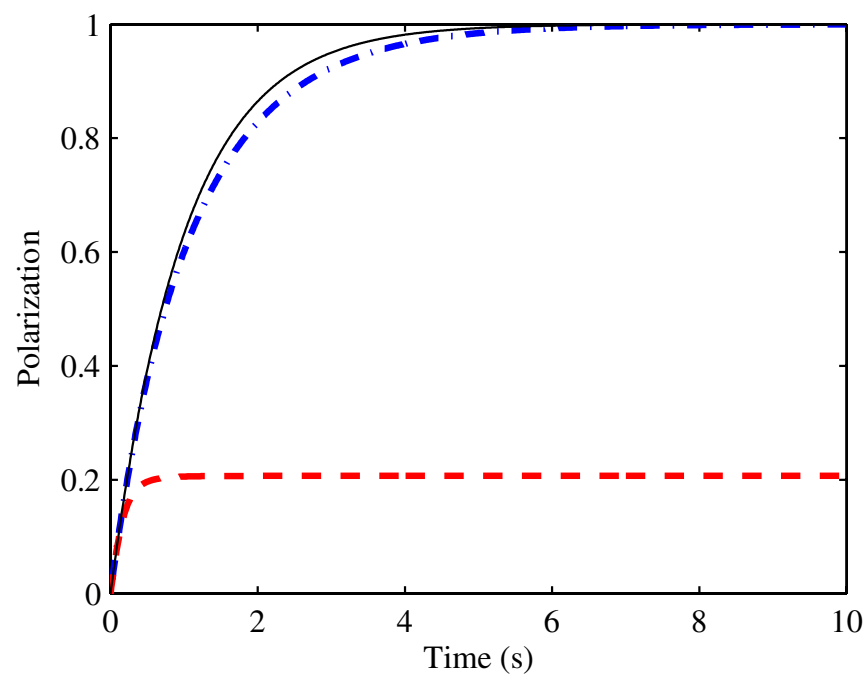

FIG. 2 (color online). Simulated polarization of 50 spins, with $R_{h}=1 \mathrm{~s}^{-1}$ and $T_{h}=0$. The dashed curve shows relaxation by noninteracting spins to a trapped state, while the dash-dotted curve corresponds to the regime in which the spin Hamiltonian efficiently equalizes the population within each eigenspace of $I_{z}$ during the relaxation. The solid curve shows exponential relaxation with rate constant $R_{h}$. exponential relaxation with rate constant $R_{h}$, as would apply if spin correlations were fully suppressed and the system could be described by a Zeeman spin temperature at all times. Finally, the dash-dotted curve is obtained by modeling the action of the many-body secular spin Hamiltonian $H_{\text {spin }}$ as instantaneously equalizing the populations of all states within a given $I_{z}$ eigenspace at the end of each $0.01 \mathrm{~s}$ time step of the simulation; during the time step, transitions between populations occur at rates determined by the superoperator for resonator-induced relaxation [9], with the interactions governed by $H_{\text {spin }}$ not explicitly included. Note that the prediction of this model is very similar to the limit (solid curve) of enforced independent spin relaxation. This is so in spite of the fact that, for $N=50$, the number of manifolds having small $I$ is vastly larger than the number having $I$ near $N / 2$.

After polarizing the spins, the resonator serves as a sensitive detector of transverse magnetization. The BOOMERANG scheme for force-detected NMR spectroscopy $[3,4]$, as with sensitive two-dimensional inductive detection schemes [13], encodes a single point of the free-induction decay (FID), or other spin evolution of interest, while suspending the effect of $H_{\text {spin }}$ during the detection period in order to prolong the transient, thus optimizing signal energy. In this scheme, a conventional NMR pulse sequence is applied to the spins, which then precess freely during an initial period $t_{1}$. This FID can be terminated by spin locking the transverse dipole, which exerts a driving force on the resonator; the resulting mechanical motion is detected, and analysis of the motion yields a measurement of $\left\langle I_{x}\right\rangle\left(t_{1}\right)$. A record of the spins' time evolution is obtained by repeating this measurement for a range of values $t_{1}$, and Fourier analysis yields the NMR spectrum. Direct driving of the resonator by the applied radio frequency (rf) field during spin locking can be minimized by applying the pulses along the length of the torsional beam, so that the direct rf torque is orthogonal to that needed to drive the resonator. Additional isolation of the spin-driven component of the signal is possible by using pulsed spin locking [13,14], which allows sampling in windows that are long compared to the detector ringdown time yet preserves the transverse magnetization against dephasing by $H_{\text {spin }}$. Eddy-current heating of the magnetic cylinders by the rf pulses could be made small by fabricating them from a low-conductivity nonmetallic ferromagnetic material, such as $\mathrm{EuO}$ [15] (see [7]). Since the intended operation is at a low temperature and a high field, even sandwich magnets made of paramagnetic materials will be fully polarized. Unwanted radiation damping could be minimized by moving $\omega_{0}$ out of resonance with $\omega_{h}$ during the FID. Possible methods for shifting $\omega_{0}$ out of resonance with $\omega_{h}$ include shifting the external field, or rotating the resonator assembly by $90^{\circ}$ about the beam axis, so that the axis of the sandwich in Fig. 1 is at a right angle with the applied field. The FID would be terminated by a $\pi / 2$ pulse to temporarily store $\left\langle I_{x}\right\rangle\left(t_{1}\right)$ along $z$ while 


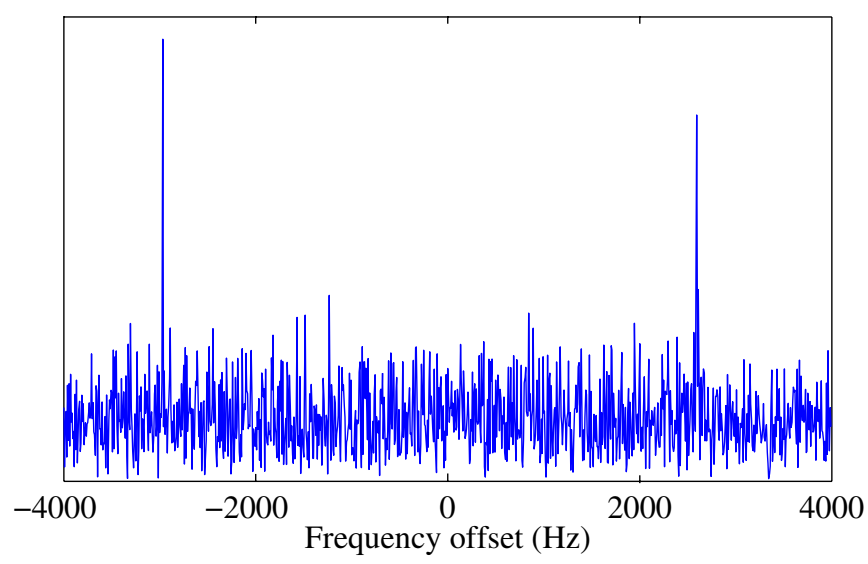

FIG. 3 (color online). Simulation of the proton NMR spectrum of a single molecule of vinyl bromide adsorbed on $\mathrm{Si}$. The noise sources are spin fluctuations, thermal motion in the resonator, and noise added during detection of the mechanical motion. A chemical shift of $0.6 \mathrm{ppm}$ and a secular dipolar coupling of $1840 \mathrm{~Hz}$ were used for the simulation. Thermal polarization was assumed at the beginning of each transient. Acquisition would require $\sim 60 \mathrm{~h}$.

the resonance condition $\omega_{h}=-\omega_{0}$ is restored, and the spin dipole would then be rotated to the transverse plane and detected by spin locking.

Several schemes exist for sensitive detection of nanomechanical motion at cryogenic temperatures. A scheme consistent with the resonator design couples the motion capacitively to a single-electron transistor. Detection of translational mechanical motion using a single-electron transistor has been demonstrated experimentally at a level of sensitivity near the quantum limit [16]. This scheme might be adapted for detection of torsional motion by capacitively coupling electrodes to the magnetic sandwich so that the gate capacitance is modulated by the mechanical motion (see [7] for detector geometries). For purposes of using simulations to characterize the potential utility of the resonator, we assumed the torsional motion to be detected with sensitivity comparable to that of a recent single-electron-transistor measurement of nanoresonator motion [16]. Specifically, we assumed that the noise torque added by the motion detector has a spectral density 32 times that of the thermal noise torque acting on the resonator in the limit $T_{h} \rightarrow 0$ [17] or, equivalently, 30 times that of the thermal noise torque at the design temperature of $T_{h}=10 \mathrm{mK}$. Note, however, that the estimated single-spin displacement signal for the example resonator described in Ref. [7] is $2 \times 10^{-16} \mathrm{~m}$, and that sensitive detection of this signal constitutes a significant experimental challenge.

As an example application, Fig. 3 shows a simulated two-proton spectrum that includes noise from spin fluctuations, thermal motion in the resonator, and noise added by the motion detector, with the latter contribution dominating. The sample is a single molecule of vinyl bromide adsorbed on $\mathrm{Si}$, a species obtained by room-temperature adsorption of dibromoethylene on Si [18]. Standard bond angles and lengths were used to estimate the Hamiltonian for dipole-dipole coupling, and a chemical-shift difference of $0.6 \mathrm{ppm}$ between the two protons was assumed [19]. A noiseless FID was simulated by using GAMMA [12], and the magnitude of the noise to be added to the FID was calculated by using the parameters for an example resonator that has a magnetic sandwich of diameter $55 \mathrm{~nm}$ and height $105 \mathrm{~nm}$, with a separation of $25 \mathrm{~nm}$ between the magnets (see [7] for details of the calculation). The resonator's field at the spins was simulated by using finiteelement software (MAXWELL 3D v11, Ansoft Corporation, Pittsburgh). Within a cylindrical volume of diameter $2 \mathrm{~nm}$ and height $1.4 \mathrm{~nm}$, the Larmor frequency differs by no more than $25 \mathrm{kHz}$ from its value at the center of the sandwich. The calculated time constant for resonatorinduced polarization between transients is $1 / R_{h}=0.8 \mathrm{~s}$, with a single-shot signal-to-noise ratio of 0.06 for detection of a single spin. Acquisition of the spectrum shown in Fig. 3 would require $\sim 60 \mathrm{~h}$, assuming the detection period for each transient lasts $3 T_{1 \rho}=3 \mathrm{~s}$ and the delay between transients is $3 / R_{h}$. Although this simulation characterizes performance that would require considerable effort to achieve experimentally, it suggests the possibility of spectroscopic studies of low-temperature samples with singleproton sensitivity.

*mcbutler@berkeley.edu

[1] J. A. Sidles et al., Rev. Mod. Phys. 67, 249 (1995).

[2] C. L. Degen et al., Proc. Natl. Acad. Sci. U.S.A. 106, 1313 (2009).

[3] G. M. Leskowitz, L. A. Madsen, and D. P. Weitekamp, Solid State Nucl. Magn. Reson. 11, 73 (1998).

[4] L. A. Madsen, G. M. Leskowitz, and D. P. Weitekamp, Proc. Natl. Acad. Sci. U.S.A. 101, 12804 (2004).

[5] K. W. Eberhardt et al., Angew. Chem., Int. Ed. 47, 8961 (2008).

[6] P. L. Kuhns et al., Phys. Rev. B 35, 4591 (1987).

[7] See supplementary material at http://link.aps.org/ supplemental/10.1103/PhysRevLett.105.177601 for additional details.

[8] B. W. Shore and P. L. Knight, J. Mod. Opt. 40, 1195 (1993).

[9] S. Haroche, in New Trends in Atomic Physics, Proceedings of the Les Houches Summer School, Session XXXVIII, edited by G. Grynberg and R. Stora (Elsevier Science, Amsterdam, 1984), pp. 195.

[10] C. L. Degen et al., Phys. Rev. Lett. 100, 137601 (2008).

[11] R. H. Dicke, Phys. Rev. 93, 99 (1954).

[12] S. A. Smith et al., J. Magn. Reson., Ser. A 106, 75 (1994).

[13] D. P. Weitekamp, Adv. Magn. Reson. 11, 111 (1983).

[14] W. K. Rhim, D. P. Burum, and D. D. Elleman, Phys. Rev. Lett. 37, 1764 (1976).

[15] J. Lettieri et al., Appl. Phys. Lett. 83, 975 (2003).

[16] M. D. LaHaye et al., Science 304, 74 (2004).

[17] A. A. Clerk, Phys. Rev. B 70, 245306 (2004).

[18] X. J. Zhou et al., Surf. Sci. 543, L668 (2003).

[19] T. Koerner et al., J. Am. Chem. Soc. 120, 5628 (1998). 